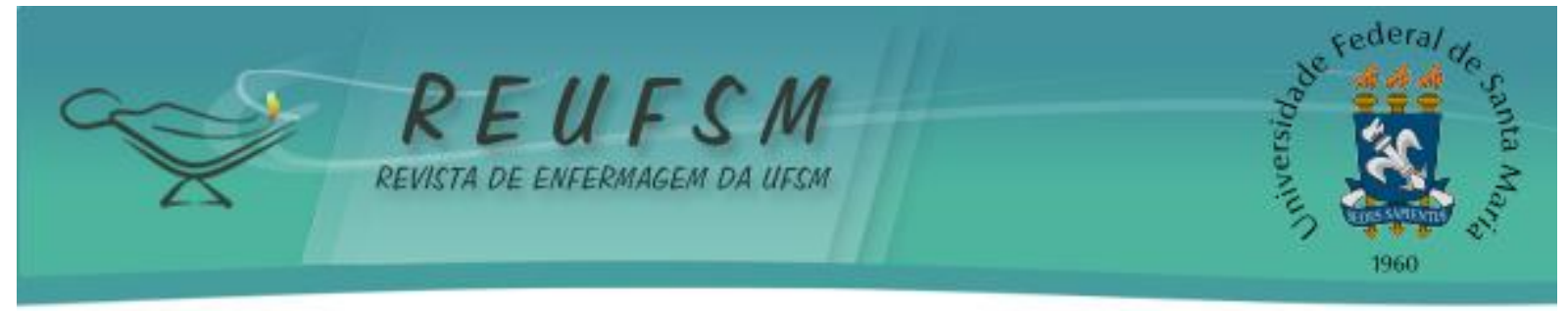

ARTIGO ORIGINAL

\title{
PREVALÊNCIA DE INFECÇÃO HOSPITALAR EM UNIDADE DE TERAPIA INTENSIVA - UM ESTUDO RETROSPECTIVO
}

\author{
PREVALENCE OF NOSOCOMIAL INFECTION IN INTENSIVE CARE UNIT - A RETROSPECTIVE \\ STUDY
} PREVALENCIA DE LA INFECCIÓN NOSOCOMIAL EN LA UNIDAD DE CUIDADOS INTENSIVOS -
UN ESTUDIO RETROSPECTIVO

Reginaldo Passoni dos Santos ${ }^{1}$

Lília Regina Mariano ${ }^{2}$

Luciane da Silva Takahashi ${ }^{3}$

Marilis de Fátima Erdmann ${ }^{4}$

Doi: $10.5902 / 2179769211233$

RESUMO: Objetivo: identificar a prevalência de infecção hospitalar em Unidade de Terapia Intensiva para adultos. Método: pesquisa quantitativa, descritiva e retrospectiva, realizada através de investigação em registros da comissão de controle de infecção hospitalar de um hospital público do interior do Paraná. A amostragem foi composta por todos os registros referentes ao período de janeiro a dezembro de 2012. A análise dos dados ocorreu utilizando-se o programa Microsoft Windows Excel ${ }^{\circledR}$, versão 2010. Resultados: a prevalência de infecção hospitalar foi de $27,35 \%$, sendo Acinetobacter sp. $(22,92 \%)$, o patógeno mais identificado. Quanto à topografia, constatou-se que as infecções do trato respiratório foram as mais frequentes, ocorrendo em $36,46 \%$ dos casos. Conclusão: 0 levantamento dos dados apontou prevalência mediana de infecção hospitalar, quando comparados ao disposto na literatura.

Descritores: Enfermagem, Prevalência; Infecção hospitalar; Unidades de terapia intensiva.

ABSTRACT: Aim: To identify the prevalence of nosocomial infection in Intensive Care Unit for adults. Method: quantitative, descriptive and retrospective study performed by research in the records of the hospital infection control committee in a public hospital in a city of Paraná. The sample comprised all records for the period January to December 2012. Data analysis occurred using Microsoft Windows Excel $\circledast$ software, version 2010. Results: The prevalence of nosocomial infection was $27.35 \%$, and Acinetobacter $s p$. (22.92\%) was the most commonly identified pathogen. In terms of topography, we found that respiratory tract infections were the most frequent, occurring in $36.46 \%$ of cases. Conclusion: The data survey showed a median prevalence of nosocomial infection when compared to the provisions in the literature.

Descriptors: Nursing, Prevalence; Cross infection; Intensive care units.

RESUMEN: Objetivo: Identificar la prevalencia de la infección nosocomial en la unidad de cuidados intensivos para adultos. Método: Estudio cuantitativo, descriptivo y retrospectivo realizado mediante investigación en los registros de la comisión de control de infección hospitalaria en un hospital público de Paraná (Brasil). La muestra fue

\footnotetext{
${ }^{1}$ Graduado em Enfermagem pela Pontifícia Universidade Católica do Paraná (PUCPR), Toledo, PR, Brasil. Email: regi-pas@hotmail.com

${ }^{2}$ Graduada em Enfermagem pela PUCPR, Toledo, PR, Brasil. E-mail: liavolgere@yahoo.com.br

3 Enfermeira. Especialista em Terapia Intensiva. Docente do Curso de Graduação em Enfermagem da PUCPR, Toledo, PR, Brasil. E-mail: luciane.takahashi@pucpr.br

${ }^{4}$ Enfermeira. Especialista em Controle de Infecção Hospitalar. Docente do Curso de Graduação em Enfermagem da PUCPR, Toledo, PR, Brasil. E-mail: maliris.erdmann@pucpr.br
} 


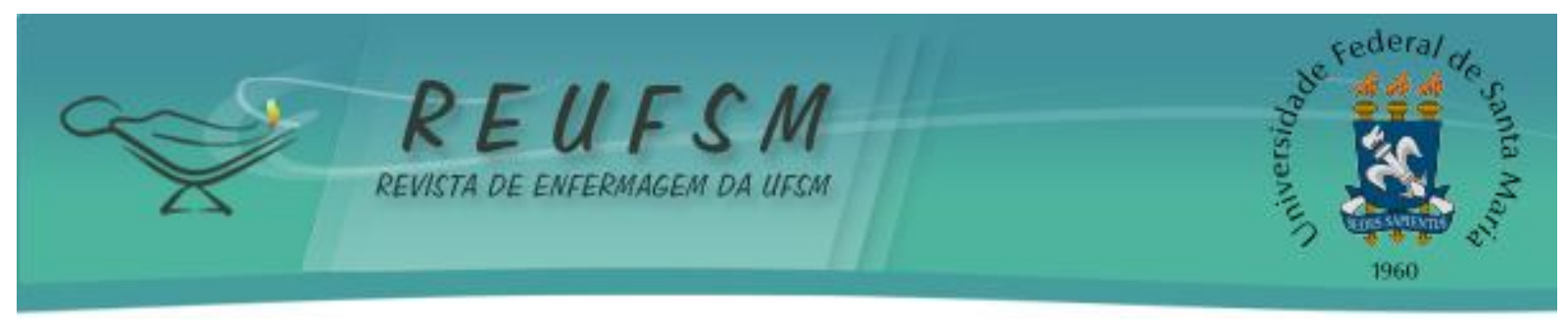

compuesta por todos los registros del período entre enero y diciembre de 2012. El análisis de datos se realizó utilizando Microsoft Windows Excel®, versión 2010. Resultados: La prevalencia de infección nosocomial fue 27,35\%, siendo Acinetobacter sp. $(22,92 \%)$ el agente patógeno más frecuente. En cuanto a la topografía, se constató que las infecciones de las vías respiratorias fueron las más frecuentes, ocurriendo en 36,46\% de los casos. Conclusión: Los datos del estudio mostraron una prevalencia mediana de la infección nosocomial, en comparación con lo dispuesto en la literatura.

Descriptores: Enfermería, Prevalencia; Infección hospitalaria; Unidades de cuidados intensivos.

\section{INTRODUÇÃO}

De acordo com a portaria $n^{\circ} 2.616 / 98$, do Ministério da Saúde (MS), infecção hospitalar (IH) ou nosocomial é definida como sendo toda infecção adquirida em ambiente hospitalar que se manifesta após a internação, durante a internação ou até mesmo após a alta hospitalar. ${ }^{1}$ Os fatores de risco para IH relacionam-se com as condições imunológicas do indivíduo, métodos invasivos de tratamento e o ambiente de exposição. ${ }^{2}$ Nesse contexto, torna-se evidente que pacientes criticamente enfermos apresentam maior vulnerabilidade a adquirirem $\mathrm{IH}$. Além de estarem em um ambiente totalmente suscetível, podem apresentar debilidade imunológica. Estes fatores podem contribuir fortemente não apenas para o alto índice de IH em Unidade de Terapia Intensiva (UTI), mas também para a alta prevalência de morbimortalidade, sendo necessário que a equipe de enfermagem e saúde sejam detentoras de conhecimentos indispensáveis sobre o controle de infecções..$^{3-4}$

O diagnóstico de infecção hospitalar é feito com base em alguns critérios clínicos que servem como norteadores. Nessa direção, a premissa inicial para que um caso de infecção seja considerado nosocomial é averiguar se as manifestações clínicas iniciaram há, no mínimo, 72 horas após sua admissão hospitalar. É fundamental saber se houve algum procedimento diagnóstico e/ou terapêutico durante este período. De acordo com a Agência Nacional de Vigilância Sanitária (ANVISA), os critérios ainda devem incluir evidências clínicas (sinais e sintomas), resultados de exames laboratoriais (microbiológicos, histopatológicos e sorológicos) e estudos de imagem (ultrassonografia, radiológicos, endoscópios, etc.). ${ }^{5}$

Partindo da ideia de que muitas das infecções hospitalares são evitáveis, e seguindo tendências mundiais no que diz respeito à prevenção de $\mathrm{IH}$, o governo brasileiro aprovou no ano de 1997 a Lei 9.431, tornando então obrigatório a implantação da Comissão de Controle de Infecção Hospitalar $(\mathrm{CClH})$ nas instituições hospitalares do país. ${ }^{6}$ Existem duas categorias de membros que compõem a $\mathrm{CCIH}$, os membros consultivos - que são profissionais representativos dos serviços de saúde (medicina, enfermagem, farmácia, laboratório de microbiologia e administração) - e os membros executores, que são os responsáveis pela implantação, execução, acompanhamento e avaliação dos programas de controle de $\mathrm{IH}$. Preconiza-se que um dos membros executores seja, preferencialmente, enfermeiro. ${ }^{1}$ Dessa forma, mesmo estando diante da premissa de interdisciplinaridade profissional e do trabalho em equipe, com frequência o enfermeiro representa ser o "carro-chefe" para a realização de atividades a serem desenvolvidas pelos membros executores. ${ }^{7}$

Diante disso, entende-se que a preocupação com o conhecimento das características epidemiológicas do cenário em que atua faz do enfermeiro um profissional que demonstra competência e conhecimento técnico-científico, características essenciais para o planejamento das atividades em sua prática diária. Nessa direção, a questão norteadora da presente pesquisa é: Qual a prevalência de IH em UTI para adultos de um hospital público do Oeste do Paraná? Com esta indagação, o objetivo primário deste estudo 


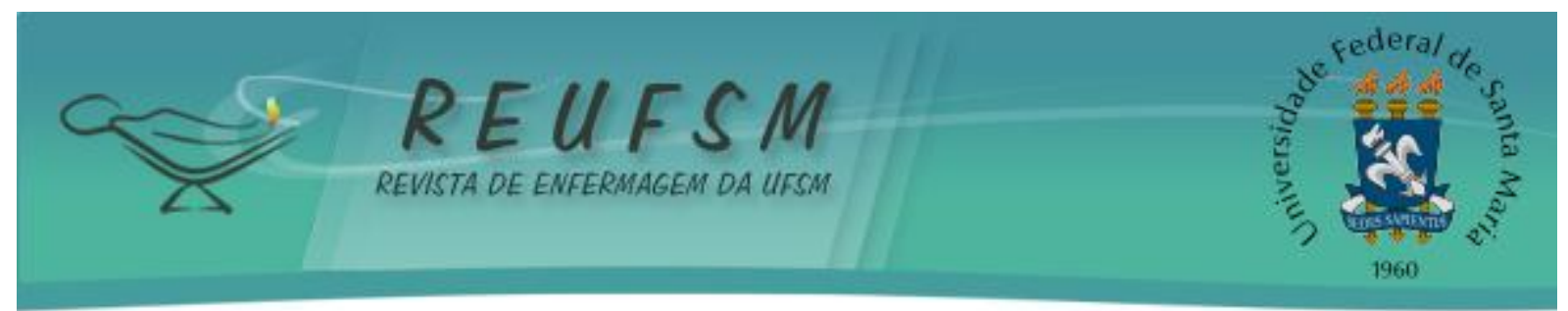

foi identificar a prevalência de IH em UTI para adultos. Como objetivo secundário, pretendeu-se levantar as temáticas de educação permanente abordadas pelos membros da $\mathrm{CCHI}$ junto a equipe de enfermagem da unidade no período pesquisado.

\section{MÉTODOS}

Pesquisa quantitativa, descritiva e retrospectiva, realizada por meio de investigação documental junto aos registros da $\mathrm{CCIH}$ na Unidade de Terapia Intensiva Adulto de um hospital público situado em um Município do interior do Paraná, o qual possui abrangência regional de atendimento à 18 Municípios e é referência em cuidados de alta complexidade em ortopedia. Quanto ao cenário de estudo, trata-se de uma UTI geral para adulto ligada ao Sistema Único de Saúde (SUS), com 12 leitos individuais cuja ocupação de vaga ocorre via Central Estadual de Leitos. A amostragem foi composta por todos os registros de notificação referentes ao período de janeiro a dezembro de 2012, excetuandose o mês de julho, pois não havia registros disponíveis relativos ao referido mês. Incluíramse registros de todos os pacientes internados na unidade no mínimo há 72 horas e que apresentaram infecção dentro do período de internação na UTI. Os dados foram coletados em agosto de 2013, por meio da utilização de fichas impressas elaboradas pelos autores com um roteiro semiestruturado, as quais serviram para o levantamento de informações acerca do número global de $\mathrm{IH}$ no período, agentes etiológicos, sítio de infecção e temáticas abordadas pela equipe da $\mathrm{CCHI}$ em ações de educação continuada realizadas junto à equipe de enfermagem da unidade, sendo estes dados obtidos junto ao livro de registros de atividades realizadas pela equipe de controle de infecção.

A tabulação ocorreu a partir da transcrição das informações contidas nas fichas para o programa Microsoft Windows Excel ${ }^{\circledR}$, versão 2010, o qual tambem serviu para realização da análise estatística descritiva e estruturação dos resultados em tabelas e gráficos, com apresentação das frequências absolutas e/ou correspondentes percentuais. Em consonância ao disposto pela Resolução 466/2012, o presente artigo teve seu projeto de pesquisa aprovado pelo Comitê de Ética em Pesquisa da Pontifícia Universidade Católica do Paraná, com emissão de parecer favorável sob o $\mathrm{n}^{\circ} 215.838$ na data de 25 de fevereiro de 2013.

\section{RESULTADOS}

Identificou-se que, de janeiro a dezembro de 2012 o hospital contabilizou 96 registros de IH na UTI para adulto, sendo que a unidade manteve no mesmo período 351 pacientes internados. Com isso, a prevalência global de $\mathrm{IH}$ foi de $27,35 \% \mathrm{com}$ uma proporção de infecção por paciente internado de quase 1:4. Das 96 notificações de IH do ano, $38(39,58 \%)$ ocorreram entre os meses de abril a junho, sendo este o trimestre com o maior número de infecção hospitalar na UTI. Com relação aos outros trimestres, o levantamento apontou que 31 (32,29\%) infecções ocorreram entre janeiro e março; 15 $(15,63 \%)$ entre julho e setembro (ressalta-se que para este trimestre, os dados relacionamse apenas aos meses de agosto e setembro); 12 (12,5\%) entre outubro e dezembro de 2012. Quanto ao número de internados $135(38,46 \%)$ pacientes foram internados entre janeiro e março; 127 (36,18\%) entre abril e junho; 34 (9,69\%) entre julho e agosto (tambem para este dado, os números referem-se apenas aos meses de agosto e setembro) e $55(15,67 \%)$ pacientes mantiveram-se internados entre outubro e dezembro (Figura 1). 

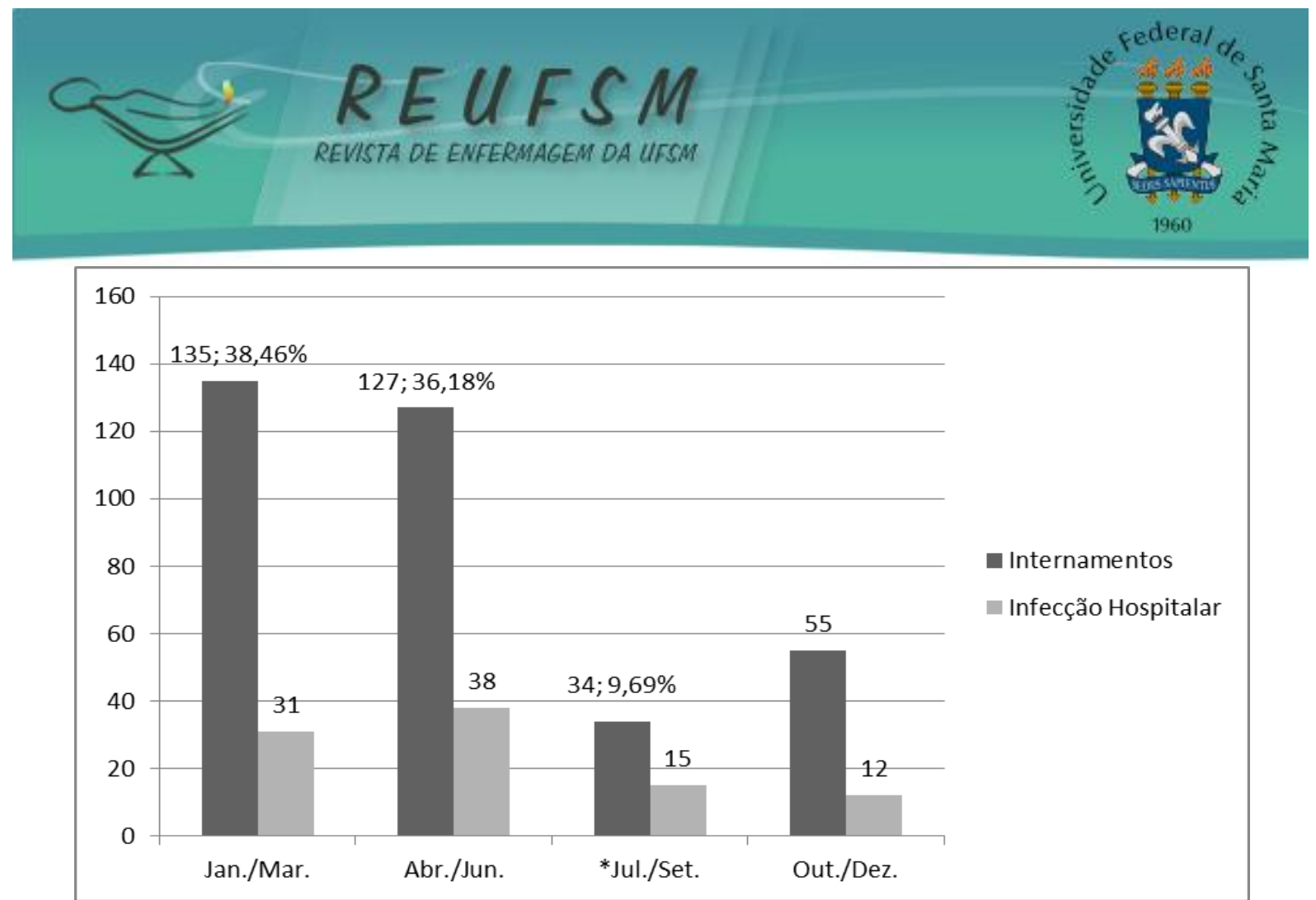

Figura 1. Distribuição do número de pacientes com infecção, segundo o número de internados por trimestre. Toledo, PR, 2012. *Dados relativos apenas aos meses de agosto e setembro.

Fonte: Registros da CCIH do hospital pesquisado.

No que diz respeito ao agente etiológico, identificou-se que $22(22,95 \%)$ infecções ocorreram por Acinetobacter sp.; 20 (20,83\%) por Pseudomonas aeruginosa; 17 (17,71\%) por outros bacilos gram-negativo (BGN); 10 (10,42\%) por Staphylococcus aureus e 27 $(28,13 \%)$ infecções tiveram outros agentes etiológicos como causa ou não havia identificação acerca do nome do microorganismo no registro (Figura 2).

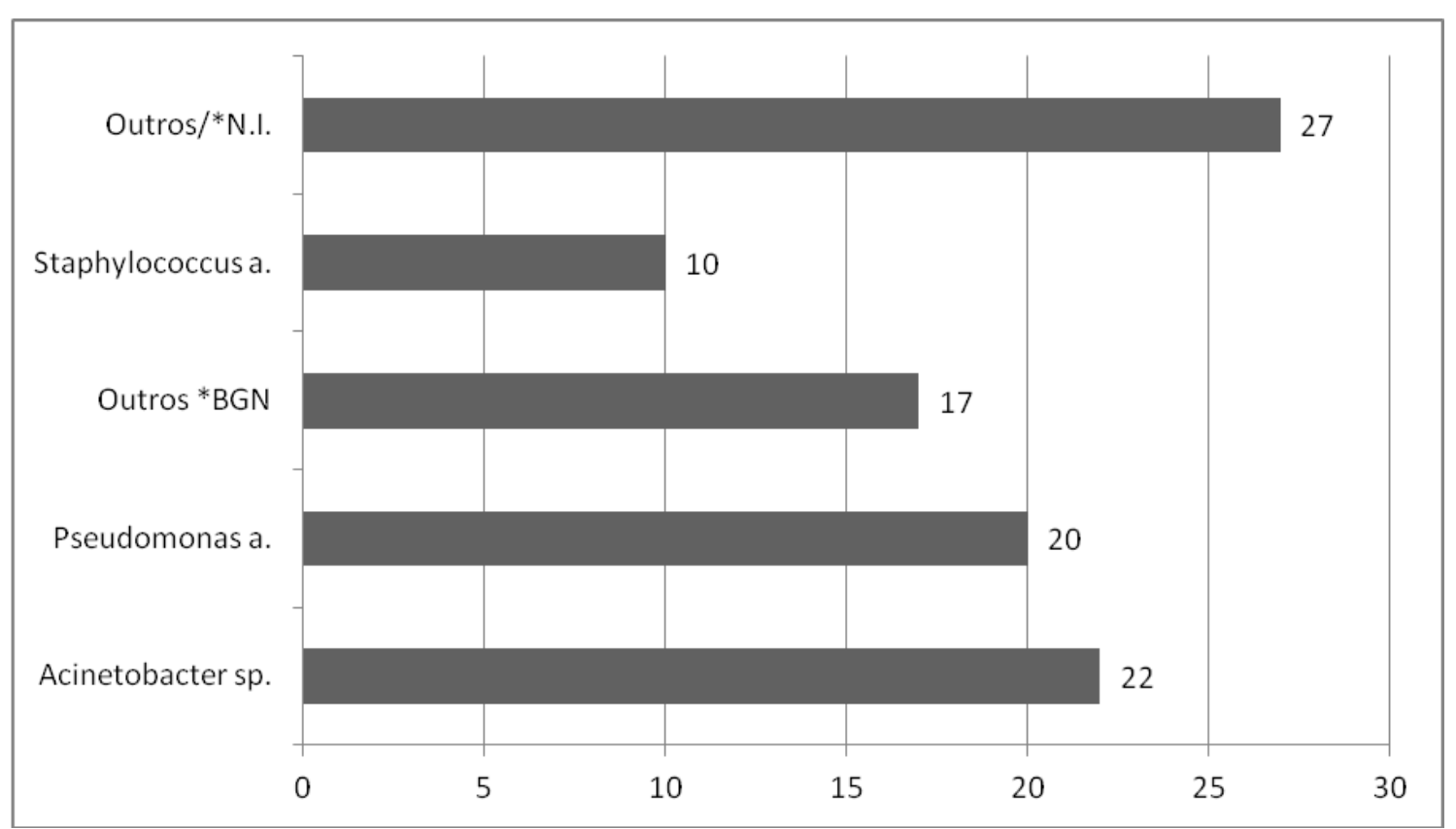

Figura 2. Distribuição do número de infecção hospitalar, segundo o agente etiológico. Toledo,PR, 2012. *N.I.: Não Identificado; BGN: Bacilo gram-negativo.

Fonte: Registros da CCIH do hospital pesquisado. 


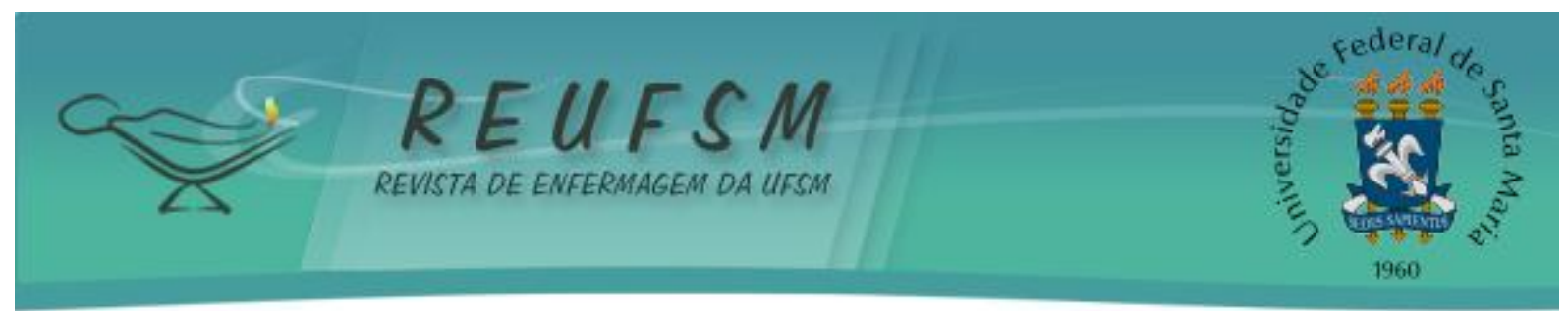

No que concerne ao sítio de infecção, $35(36,46 \%)$ registros apontaram infecção do trato respiratório (ITR); 15 (15,63\%) infecções do trato urinário (ITU); 13 (13,54\%) infecções identificadas em aspirado de líquido abdominal (LA); duas (2,08\%) em cateter venoso central (CVC); duas (2,08\%) identificadas por aspirado de líquido cefalorraquidiano (LCR) e duas (2,08\%) por aspirado de conteúdo em dreno de tórax. Nos 27 (28,13\%) registros restantes não havia identificação topográfica do sítio infectado (Figura 3).

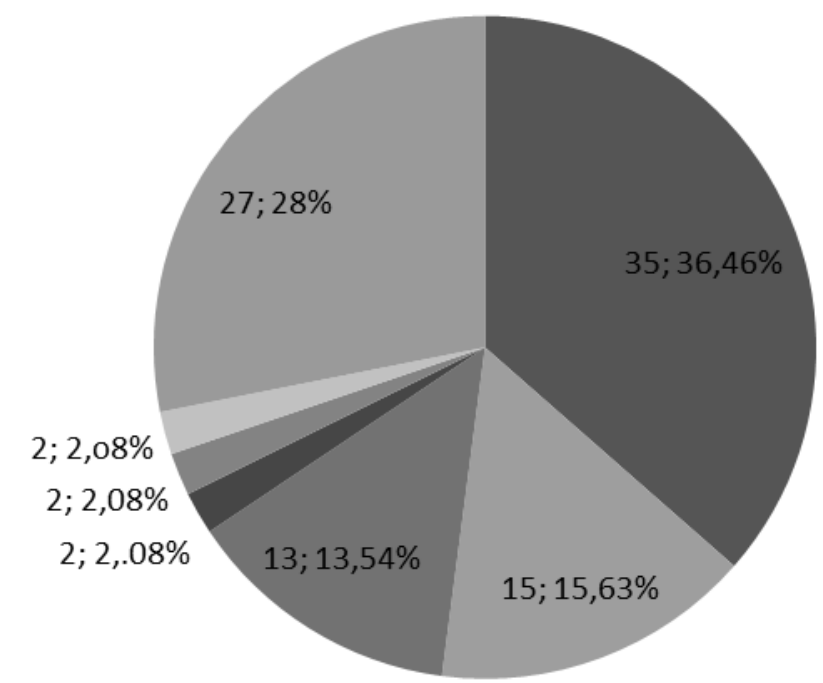

ITR $\square$ ITU $\square$ Aspirado de LA $\square$ CVC $\square$ Aspirado de LCR $\square$ Dreno de tórax $\square$ Não identificado

Figura 3. Distribuição do número de infecção, segundo o sítio. Toledo, PR, 2012.

Fonte: Registros da CCIH do hospital pesquisado.

Em relação às temáticas abordadas pela equipe da $\mathrm{CCIH}$ através do Programa de Educação Continuada (PEC) para os colaboradores de enfermagem da UTI, levantou-se que no período de janeiro a dezembro de 2012 foram realizados cursos de atualização com as seguintes pautas: precauções padrão e normas de isolamento; infecção cruzada; lavagem das mãos; monitoração da lavagem das mãos pré e pós contato com o paciente; uso de adereços/adornos; normas de biossegurança; desinfecção de superfícies. As atividades de atualização foram realizadas nos meses de janeiro, abril, julho e outubro de 2012, conforme distribuição apresentada na tabela 1. Ressalta-se que, a equipe manteve-se constante durante o período sem alta rotatividade de colaboradores na unidade.

Tabela 1 - Temáticas abordadas pela CCIH na educação continuada, segundo o mês de atividade. Toledo, PR, 2012.

\section{Ações do PEC na UTI}

Precauções padrão e normas de isolamento Infecção cruzada

Lavagem das mãos

Monitoração da higienização das mãos

Uso de adornos/adereços

Normas de biossegurança

Desinfecção de superfície

\section{Mês da atividade}

Janeiro; Julho; Outubro.

Janeiro; Abril; Julho; Outubro.

Janeiro; Abril; Julho; Outubro.

Julho.

Janeiro; Julho; Outubro.

Janeiro; Julho; Outubro.

Janeiro; Outubro.

Fonte: Registros da CCIH do hospital pesquisado. 


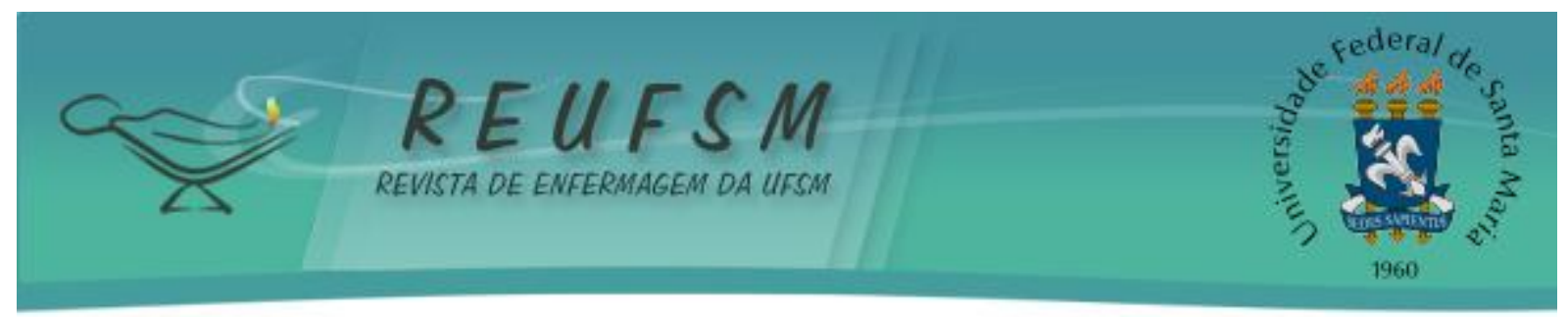

DISCUSSÃO

Diversos fatores contribuem para que o controle de IH em UTI apresente-se como um desafio à equipe de enfermagem e saúde, dentre eles citam-se a alta demanda e rotatividade de pacientes, capital humano com extensa jornada laboral, estrutura física inadequada e as dificuldades em se manter um Programa de Educação Continuada; ademais, a multirresistência dos agentes etiológicos é cada vez mais recorrente. ${ }^{8-9}$ Em estudo retrospectivo realizado no Hospital de Clínicas da Faculdade de Medicina de Ribeirão Preto (HCFMRP), identificou-se a prevalência de $12,98 \%$ de casos de pacientes em UTI com IH por bactérias multirresistentes. ${ }^{10}$ Já com relação aos números globais, em estudo ${ }^{11}$ realizado na cidade de Fortaleza (CE) levantaram uma prevalência de $\mathrm{IH}$ em $28,77 \%$ dos pacientes internados durante o período de 12 meses, ao passo que nesta pesquisa a prevalência foi de $27,35 \%{ }^{11}$ A análise realizada em um hospital universitário brasileiro, identificou IH em 13\% dos pacientes incluídos no estudo. ${ }^{12} \mathrm{Em}$ investigação prospectiva, multicêntrica e internacional ${ }^{13}$ - realizada simultaneamente, num único dia, em 75 países de todo o mundo os resultados apontaram que dos 1.235 pacientes internados em 90 UTIs brasileiras na data do estudo, a prevalência de IH foi de $61,16 \%$ dos casos. ${ }^{13}$

Tais dados trazem preocupação às equipes de saúde e administração hospitalar, tendo em vista que além de aumentar o tempo de internação do paciente/cliente, as infecções nosocomiais em unidades intensivas apresentam-se altamente onerosas. Apesar de terem identificado uma baixa prevalência de $\mathrm{IH}(8,9 \%)$ entre os pacientes investigados, estudo realizado em Minas Gerais levantaram que os pacientes que não apresentaram infecção geraram um gasto médio de $\mathrm{R} \$ 359,00$ enquanto que dentre aqueles com $\mathrm{IH}$ o gasto médio foi de $\mathrm{R} \$ 618,00$ por dia de internação em UTI, sendo que o tempo de internação em dias foi em média 5 vezes maior para estes pacientes. ${ }^{14}$

Identificou-se neste estudo a prevalência de Acinetobacter. sp. correspondendo a $22,95 \%$ do total de infecções. Em pesquisa com objetivos semelhantes aos elencados para este estudo, ${ }^{15}$ a incidência de IH por este patógeno foi de $71 \%$. Estudo ${ }^{16}$ ao levantar o perfil de resistência antimicrobiana desta bactéria em UTI, constatou a existência de cepas multirresistentes até mesmo para antibacteriano de amplo espectro. Outros patógenos frequentemente causadores de IH em UTI também foram identificados no estudo, sendo eles: Staphylococcus aureus e Pseudomonas aeruginosa. ${ }^{16}$ A infecções cruzadas transmitidas pelas mãos dos profissionais de saúde - estão entre as principais causas de contaminação por Staphylococcus, o qual apresenta significativo grau de virulência e resistência antimicrobiana. ${ }^{17-18}$ Entretanto, em estudo realizado na Índia constatou-se que o microorganismo Acinetobacter baumanni tambem apresenta alta taxa de resistência antimicrobiana e índices de mortalidade, estando relacionado principalmente com pneumonia associada à ventilação (PAV). O estudo alerta para a necessidade de uso adequado, fundamentado e racional dos diversos tipos de agentes antimicrobianos disponíveis no mercado. ${ }^{19}$

Um estudo ${ }^{8}$ retrospectivo e documental realizado em Campina Grande (PB), identificou maior prevalência de IH por Staphylococcus aureus $(14,86 \%),{ }^{8}$ superior a encontrada na presente pesquisa $(10,42 \%)$. No que diz respeito a prevalência de IH por Pseudomonas aeruginosa, evidenciou-se em um estudo que este foi o microorganismo mais identificado nas culturas realizadas, correspondendo a uma prevalência de 33,8\%, seguido por Staphylococcus aureus $(25,2 \%)$ e Acinetobacter $s p .(12,6 \%) .{ }^{11}$ Estes tambem foram os agentes patógenos mais frequentes desta pesquisa, contudo em ordem e prevalências distintas. Já com relação à resistência antimicrobiana, apontou que a sensibilidade patogênica à fármacos da classe dos beta-lactâmicos foi mais prevalente em $58 \%$ dos casos. $^{11}$ 


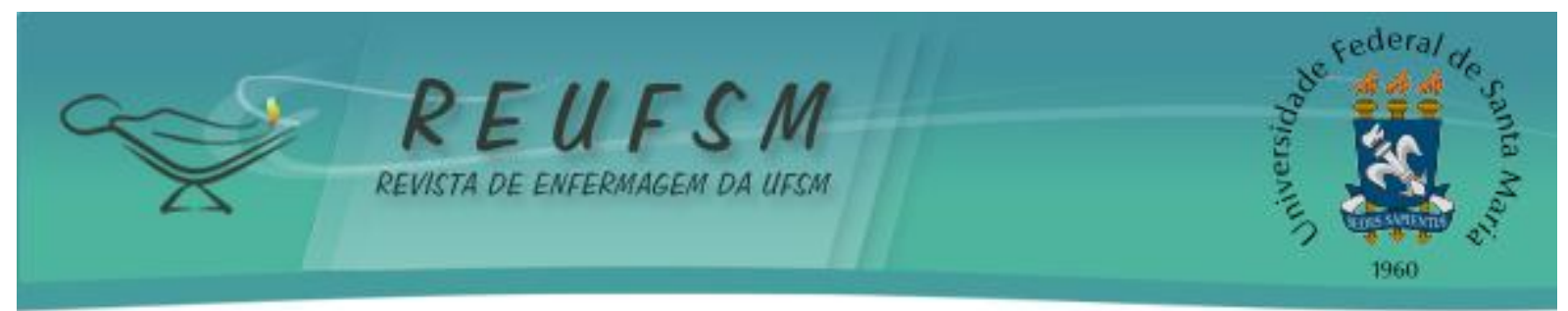

Quanto ao sítio de infecção, assim como este estudos apontam que as infecções dos tratos respiratório e urinário são identificadas com maior prevalência em pacientes hospitalizados em UTIs. ${ }^{11,18}$ Nessa direção, em pesquisa realizada num hospital universitário as infecções do trato urinário mostraram-se predominantes, correspondendo a $25,6 \%$ do total, ${ }^{12}$ prevalência maior que neste estudo a qual ficou em 15,63\% sendo as infecções do trato respiratório as mais frequentes (figura 3.).

A educação continuada dos trabalhos de UTI apresenta-se como uma das ferramentas da CCHI na tentativa de prevenção e controle das IHs. Nesse sentido, acredita-se que a instituição do PEC nas unidades hospitalares tem por objetivo garantir aos colaboradores a "[...] aquisição de conhecimento, de habilidades e de atitudes para interagir e intervir na realidade além de auxiliar e minimizar os problemas advindos da defasagem na formação". ${ }^{206619}$ Pode-se observar através do disposto na Tabela 1 , que as temáticas das atividades desenvolvidas pela equipe da CCHI junto aos colaboradores da UTI enquadram-se nas recomendações da Agência Nacional de Vigilância Sanitária (ANVISA), a qual enfatiza que a higienização das mãos apresenta-se como a principal medida para o controle da IH, o que justificaria o fato de a referida temática ter sido abordada em todos os meses de atividades da CCIH (tabela 1). ${ }^{21}$

Quanto as normas de biossegurança especificamente, apesar dos relatos de compreensão acerca da importância, muitos trabalhadores em saúde admitem não tomarem todas as medidas necessárias, principalmente, no que se refere ao uso dos Equipamentos de Proteção Individual (EPIs). ${ }^{22}$ Nesse contexto, alguns autores lembram que mesmo com um PEC permanente, a falta de adesão dos profissionais de enfermagem às medidas de prevenção e controle da IH mostra-se como um grande empecilho e condicionante desafiador aos profissionais da $\mathrm{CCIH} .{ }^{23}$

\section{CONCLUSÃO}

O levantamento dos dados epidemiológicos acerca da infecção hospitalar na unidade de terapia intensiva pesquisada apontou prevalência mediana de infecção hospitalar, quando comparados ao disposto na literatura. A educação continuada apresenta-se como importante ferramenta dentre as medidas estabelecidas pela CCHI na prevenção e controle das infecções, principalmente no que diz respeito à sensibilização por parte da equipe de enfermagem para a importância prática da higienização das mãos.

Destarte, este estudo contribuiu para o conhecimento dos dados epidemiológicos da IH dentro do contexto hospitalar da instituição pesquisada, possibilitando a equipe de saúde elencar estratégicas e medidas de prevenção com maior especificidade e direcionamento voltado à realidade local. Além disso, ratificou-se que o controle das infecções é essencial para minimização do tempo de internamento do paciente na unidade intensiva e, por conseguinte, redução dos gastos financeiros, sendo a falta de tais dados os fatores limitantes desta pesquisa. Dessa forma, sugere-se que estudos mais robustos e em realidades distintas sejam realizados, afim de angariar subsídio científico que fortaleça a prática baseada em evidências.

\section{REFERÊNCIAS}

1. Brasil. Ministério da Saúde. Gabinete do Ministro. Portaria $n^{\circ} 2.616$, de 12 de maio de 1998. Diretrizes e normas para a prevenção e o controle das infecções hospitalares. Brasília: Ministério da Saúde; 1998. 


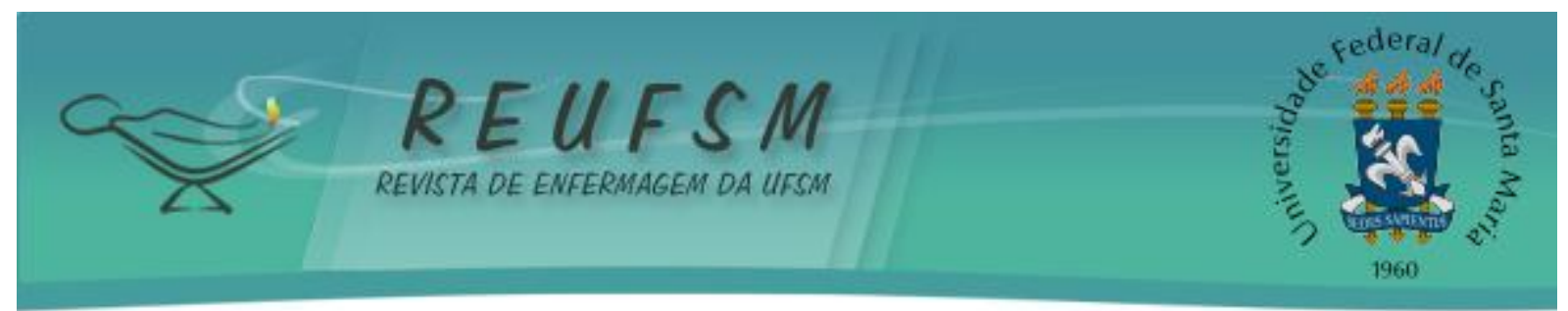

2. Lichy RF, Marques IR. Fatores de risco para infecção hospitalar em unidades de terapia intensiva: atualização e implicações para a enfermagem. Rev Enferm UNISA [Internet]. 2002 [acesso em 2012 dez 1];3:43-9. Disponível em: http: / /www.unisa.br/graduacao/biologicas/enfer/revista/arquivos/2002-09.pdf.

3. Lisboa T, Pórvoa P. Prevalência e desfechos das infecções nas UTIs brasileiras: mais uma peça no quebra-cabeça... Rev Bras Ter Intensiva [Internet]. 2012 [acesso em 2012 dez 1];24(2):115-6. Disponível em: http://www.scielo.br/pdf/rbti/v24n2/03.pdf.

4. Favarin SS, Camponogara S. Perfil dos pacientes internados na unidade de terapia intensiva adulto de um hospital universitário. Rev Enferm UFSM [Internet]. 2012 [acesso em 2013 out 25];2(2):320-9. Disponível em: http://cascavel.ufsm.br/revistas/ojs2.2.2/index.php/reufsm/article/view/5178/3913.

5. Agência Nacional de Vigilância Sanitária (Anvisa). Critérios diagnósticos de infecção relacionada à assistência à saúde. Brasília: Anvisa; 2013. (Segurança do Paciente e Qualidade em Serviços de Saúde; 2).

6. Pereira MS, Souza ACS, Tipple AFV, Prado MA. A infecção hospitalar e suas implicações para o cuidado da enfermagem. Texto \& Contexto Enferm [Internet]. 2005 [acesso em 2012 dez 1];14(2):250-7. Disponível em: http://www.scielo.br/pdf/tce/v14n2/a13v14n2.pdf.

7. Giarola LB, Baratieri T, Costa AM, Bedendo J, Marcon SS, Waidman MAP. Infecção hospitalar na perspectiva dos profissionais de enfermagem: um estudo bibliográfico. Cogitare Enferm [Internet]. 2012 [acesso em $2012 \mathrm{dez}$ 1];17(1):151-7. Disponível em: http://ojs.c3sl.ufpr.br/ojs2/index.php/cogitare/article/view/26390/17583.

8. Araújo MFM, Beserra EP, Marques MB, Moreira RAN, Araújo TM, Caetano JA. Dificuldades dos profissionais da saúde no controle de infecções hospitalares. Rev Enferm UFPE Online [Internet]. 2010 [acesso em 2013 out 10];4(2):587-95. Disponível em: http://www.revista.ufpe.br/revistaenfermagem/index.php/revista/article/viewFile/790/pdf_7.

9. Pereira MS, Prado MA, Sousa JT, Tipple AFV, Souza ACS. Controle de infecção hospitalar em unidade de terapia intensiva: desafios e perspectiva. Rev Eletrônica Enferm [Internet]. 2000 [acesso em 2013 out 10];2(1). Disponível em: http://www.revistas.ufg.br/index.php/fen/article/view/679/747.

10. Andrade D, Leopoldo VC, Haas VJ. Ocorrência de bactérias multiresistentes em um centro de terapia intensiva de hospital brasileiro de emergências. Rev Bras Ter Intensiva (RBTI) [Internet]. 2006 [acesso em 2013 out 10];18(1):27-33. Disponível em: http://www.scielo.br/pdf/rbti/v18n1/a06v18n1.pdf.

11. Barros LM, Bento JNC, Caetano JA, Moreira RAN, Pereira FGF, Frota NM, et al. Prevalência de micro-organismo e sensibilidade antimicrobiana de infecções hospitalares em unidade de terapia intensiva de hospital público no Brasil. Rev Ciênc Farm Básica Apl [Internet]. 2012 [acesso em 2013 out 10];33(3):429-35. Disponível em: http://servbib.fcfar.unesp.br/seer/index.php/Cien_Farm/article/viewFile/2211/1267.

12. Oliveira AC, Kovner CT, Silva RS. Infecção hospitalar em unidade de terapia intensiva de um hospital universitário brasileiro. Rev Latinoam Enferm [Internet]. 2010 [acesso em 2013 out 12];18(2):[8 telas]. Disponível em: http://www.scielo.br/pdf/rlae/v18n2/pt_14.pdf.

13. Silva E, Dalfior Junior L, Fernandes HS, Moreno R, Vicent JL. Prevalência e desfechos clínicos de infecções em UTIs brasileiras: subanálise do estudo EPIC II. Rev Bras Ter Intensiva [Internet]. 2012 [acesso em 2013 out 12];24(2):143-50. Disponível em: http://www.scielo.br/pdf/rbti/v24n2/08.pdf. 


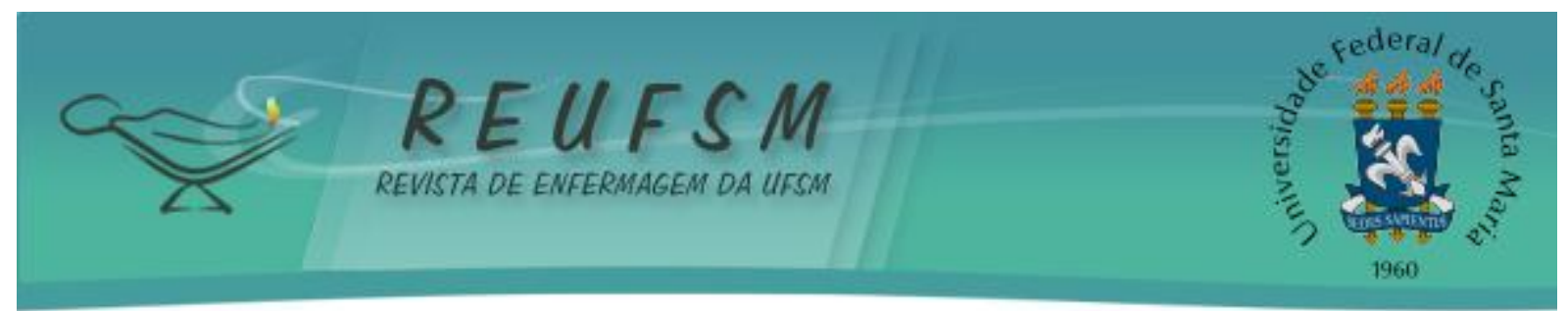

14. Nangino GO, Oliveira CD, Correia PC, Machado NM, Dias ATB. Impacto financeiro das infecções nosocomiais em unidades de terapia intensiva de hospital filantrópico de Minas Gerais. [Internet]. 2012 [acesso em 2013 out 12];24(4):357-61. Disponível em: http://www.scielo.br/pdf/rbti/v24n4/a11v24n4.pdf.

15. Henes MA, Silva SC, Fornari JV, Barnabé AS, Ferraz RRN. Incidência de infecção por Acinetobacter em uma unidade de tratamento especial de um hospital público do Estado de São Paulo. Science in Health [Internet]. 2013 [acesso em 2013 out 20];4(2):97-101. Disponível em: http://arquivos.cruzeirodosuleducacional.edu.br/principal/new/revista_scienceinhealth/11_maio _ago_2013/Science_04_02_97-101.pdf.

16. Pontes VMO, Menezes EA, Cunha FA, Ângelo ARF, Salviano MNC, Oliveira IRN. Perfil de resistência de Acinetobacter baumannii a antimicrobianos nas unidades de terapia intensiva e semi-intensiva do Hospital Geral de Fortaleza. Rev Bras Anal Clin [Internet]. 2006 [acesso em 2013 out 12];38(2):123-6. Disponível em: http://www.sbac.org.br/pt/pdfs/rbac/rbac_38_02/rbac3802_12.pdf.

17. Catão RMR, Silva PMF, Feitosa RJP, Pimentel MC, Pereira HS. Prevalência de infecções hospitalares por Staphylococcus aureus e perfil de suscetibilidade aos antimicrobianos. Rev Enferm UFPE Online [Internet]. 2013 [acesso em 2013 out 25];7(8):5257-64. Disponível em: http://www.revista.ufpe.br/revistaenfermagem/index.php/revista/article/view/4254/pdf_325.

18. Borges LFA. Higiene das mãos de profissionais de saúde em um hospital brasileiro: adesão, controle de infecção e transmissão de Staphylococcus aureus [tese]. Uberlândia: Universidade Federal de Uberlândia, Instituto de Ciências Biomédicas; 2009. 67 p.

19. Bhaskar T, Preetinder S, Sanjay A, Veena A. Profile of infection microorganisms causing ventilator-associated pneumonia: a clinical study from recourse limited intensive care unit. J Anesthesiology Clin Pharmacol [Internet]. 2013 [acesso em 2014 jan 15];29(3):361-6. Disponível em: http://joacp.org/temp/JAnaesthClinPharmacol293361-5222277_143022.pdf.

20. Bezerra ALQ, Queiroz ES, Weber J, Munari DB. O processo de educação continuada na visão de enfermeiros de um hospital universitário. Rev Eletrônica Enferm [Internet]. 2012 [acesso em 2013 out 25];14(3):618-25. Disponível em: http://www.fen.ufg.br/fen_revista/v14/n3/pdf/v14n3a19.pdf.

21. Agência Nacional de Vigilância Sanitária (Anvisa). Medidas de prevenção de infecção relacionada à assistência à saúde. Brasília: Anvisa; 2013. (Série Segurança do Paciente e Qualidade em Serviços de Saúde; 4).

22. Gallas SR, Fontana RT. Biossegurança e a enfermagem nos cuidados clínicos: contribuições para a saúde do trabalhador. Rev Bras Enferm [Internet]. 2010 [acesso em 2013 out 25];63(5):786-92. Disponível em: http://www.scielo.br/pdf/reben/v63n5/15.pdf.

23. Silva EFF, Chrizostimo MM, Azevedo SL, Souza DF, Braga LAS, Lima JL. Um desafio para o controlador de infecção: falta de adesão da enfermagem às medidas de prevenção e controle. Enferm Glob [Internet]. 2013 [acesso em 2013 out 25];12(31):316-56. Disponível em: http://revistas.um.es/eglobal/article/viewFile/149491/150071.

Data de recebimento: $30 / 10 / 2013$

Data de aceite: $21 / 03 / 2014$

Contato com autor responsável: Reginaldo Passoni dos Santos

Endereço Postal: Rua Getúlio Vargas, 574, Boa Esperança, Toledo/PR, Brasil. CEP: 85909-110.

E-mail: regi-pas@hotmail.com 